\title{
Hammett spectral correlations in benzofuranyl flavonols
}

\author{
G. Thirunarayanan ${ }^{1, \star}$, K. G. Sekar ${ }^{2}$ \\ ${ }^{1}$ Department of Chemistry, Annamalai University, Annamalainagar - 608002, Tamil Nadu, India \\ ${ }^{2}$ Department of Chemistry, National College, Tiruchirrappalli - 620001, Tamil Nadu, India \\ *E-mail address: drgtnarayanan@gmail.com
}

\begin{abstract}
A series containing eleven benzofuranyl flavonols have been prepared by cyclization of 3hydroxybenzofuranyl chalcones with $30 \%$ hydrogen peroxide in the presence of sodium bicarbonate. The synthesized flavonols were characterized by their physical constants, analytical and spectroscopic data. The infrared spectral $v \mathrm{OH}, \mathrm{CO}$ stretches $\left(\mathrm{cm}^{-1}\right)$, NMR chemical shifts of $\mathrm{OH}, \mathrm{CO}(\delta, \mathrm{ppm})$ of these flavonols were assigned and correlated with Hammett substituent constants, $\mathrm{F}$ and $\mathrm{R}$ parameters using single and multi linear regression analysis. From the results of statistical analyses, the effects of substituents on the above group frequencies were discussed.
\end{abstract}

Keywords: Benzofuranyl flavonols; Hammett equation; IR spectra; NMR spectra; Regression analysis

\section{INTRODUCTION}

Hammett spectral correlation is useful for predicting the ground state equilibration of organic molecules such as unsaturated carbonyl compounds [1], alkenes [2], polyenes [3], acyl halides and its esters [4]. Infrared spectra are a good tool for analyzing the structure of organic molecules, qualitative and quantitative measurements, $s$-cis and $s$-trans equilibration of unsaturated ketones, anti- and gauche- form of acyl halides and esters [4,5]. The NMR spectroscopy was utilized for predicting spatial arrangements of the $E$ or $Z$ isomers and conformers in unsaturated ketones, pyrazolines and it derivatives [6,7]. The effect of substituents on the above molecular conformers and isomers also studied through Hammett substituent constants, F and $\mathrm{R}$ parameters [8]. Thirunarayanan have studied the effect of substituents on spectral frequencies in thiadiazole-2-amines [9].

Joseph et al have studied the effect of substituents on dimethyl phenyl chalcones by Hammett equation with spectral data [10]. Sekar and Thirunarayanan have studied the spectral correlation in pyrazoline derivatives [11]. The substituent effects on oxazine-2amines were studied using spectral data using single and multi-regression analysis [12]. Kamalakkannan et al have studied the substituent effects on 2-indolyl chalcones by electrochemical redox potential with Hammett equation [13].

Within the above view there is no report available for the study of effect of substituents on the 3-hydroxy-2-(substituted phenyl)-4H-pyrano[3,2-b][1] benzofuron-4-ones through spectral data using regression analysis. 
Therefore the authors have taken effort to prepare some 3-hydroxy-2-(substituted phenyl)-4H-pyrano[3,2-b][1]benzofuron-4-one derivatives and recorded the infrared and NMR spectra for studying the effect of substituents on the spectral frequencies.

\section{EXPERIMENTAL}

\section{1. General}

All chemicals and solvents used in this present study were procured from SigmaAldrich and Merck companies. The infrared spectra of all oxazine imines have been recorded in SHIMADUZ Fourier Transform IR spectrophotometer using $\mathrm{KBr}$ disc. The NMR spectra of all compounds were recorded in BRUKER AV 400 type spectrometer, using $\mathrm{CDCl}_{3}$ as a solvent, $400 \mathrm{MHz}$ frequency was applied for recording ${ }^{1} \mathrm{H}, 100 \mathrm{MHz}$ for ${ }^{13} \mathrm{C}$ NMR spectra, taking TMS as standard.

\section{2. General procedure for synthesis of 3-hydroxy-2-(substituted phenyl)-4H-pyrano [3, 2-b][1]benzofuron-4-one derivatives}

About $2 \mathrm{mmol}$ of 3-hydroxybenzofuranyl chalcones, $5 \mathrm{~mL}$ of $5 \%$ sodium bicarbonate, $10 \mathrm{~mL}$ of ethanol were vigorously stirred in the three necked flask under cooling condition (Scheme 1). To this reaction mixture, added drop-wise $5 \mathrm{~mL} 30 \%$ hydrogen peroxide through dropping funnel for half an hour duration and continued the stirring to one hour and it was kept overnight.

The mixture was diluted with $20 \mathrm{~mL}$ of water and acidified with concentrated $\mathrm{H}_{2} \mathrm{SO}_{4}$ (5-7 mL). The obtained solid product was filtered at the pump washed with water, dried and recrystallized with ethanol. The analytical, physical constants and spectroscopic data of synthesized substituted flavonols were presented in Table 1.

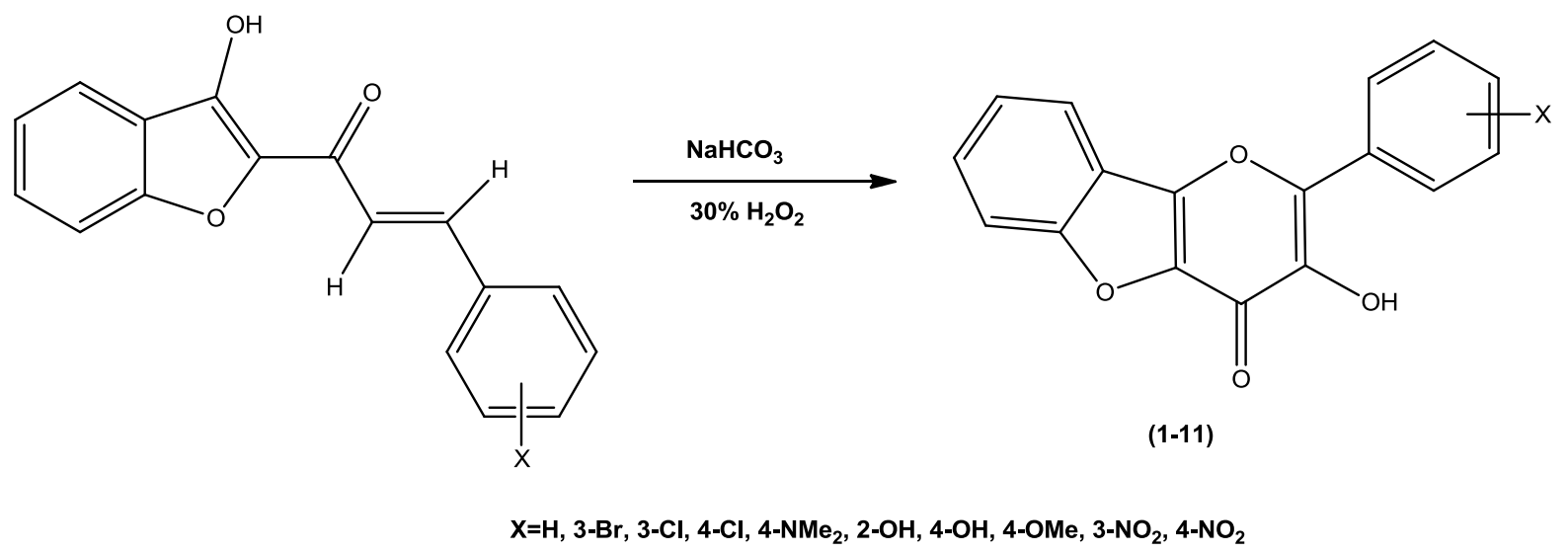

Scheme 1. Synthesis of 3-hydroxy-2-(substituted phenyl)-4H-pyrano[3,2-b][1]benzofuron-4-ones.

\section{RESULTS AND DISCUSSION}

In the present study, the author have investigated the effect of substituent on spectral frequencies of 3-hydroxy-2-(substituted phenyl)-4H-pyrano[3,2-b][1]benzofuron-4-ones 
using Hammett substituent constants, F and R parameters by linear regression analysis [1519].

Table 1. Analytical, physical constants and spectroscopic data of 3-hydroxy-2-(substituted phenyl)$4 H$-pyrano[3,2-b][1]benzofuron-4-ones.

\begin{tabular}{|c|c|c|c|c|c|c|c|c|c|}
\hline \multirow{2}{*}{ Entry } & \multirow{2}{*}{$\mathbf{X}$} & \multirow{2}{*}{ M.F. } & \multirow{2}{*}{ M.W. } & \multirow{2}{*}{$\begin{array}{l}\text { m.p. } \\
\left({ }^{\circ} \mathrm{C}\right)\end{array}$} & \multirow{2}{*}{$\begin{array}{l}\text { Mass } \\
(\mathrm{m} / \mathbf{z})\end{array}$} & \multicolumn{2}{|c|}{$\begin{array}{c}\text { IR } \\
\left(v, \mathbf{c m}^{-1}\right)\end{array}$} & \multicolumn{2}{|c|}{$\begin{array}{c}\text { NMR } \\
(\delta, p p m)\end{array}$} \\
\hline & & & & & & $\mathrm{OH}$ & $\mathrm{CO}$ & $\mathrm{OH}$ & $\mathrm{CO}$ \\
\hline 1 & $\mathrm{H}$ & $\mathrm{C}_{18} \mathrm{H}_{12} \mathrm{O}_{4}$ & 278 & $112-113$ & $278\left[\mathrm{M}^{+}\right]$ & 3410 & 1689 & 7.143 & 171.26 \\
\hline 2 & $3-\mathrm{Br}$ & $\mathrm{C}_{18} \mathrm{H}_{11} \mathrm{BrO}_{4}$ & 341 & $138-139$ & $\begin{array}{l}341\left[\mathrm{M}^{+}\right], \\
343\left[\mathrm{M}^{2+}\right]\end{array}$ & 3414 & 1689 & 7.032 & 170.26 \\
\hline 3 & $3-\mathrm{Cl}$ & $\mathrm{C}_{18} \mathrm{H}_{11} \mathrm{ClO}_{4}$ & 297 & $127-128$ & $\begin{array}{l}297\left[\mathrm{M}^{+}\right], \\
299\left[\mathrm{M}^{2+}\right]\end{array}$ & 3413 & 1690 & 7.101 & 170.32 \\
\hline 4 & $4-\mathrm{Cl}$ & $\mathrm{C}_{18} \mathrm{H}_{11} \mathrm{ClO}_{4}$ & 297 & $\begin{array}{c}144-145 \\
(142-144) \\
{[14]}\end{array}$ & $\begin{array}{l}297\left[\mathrm{M}^{+}\right], \\
299\left[\mathrm{M}^{2+}\right]\end{array}$ & 3413 & 1690 & 7.132 & 170.03 \\
\hline 5 & $4-\mathrm{NMe}_{2}$ & $\mathrm{C}_{20} \mathrm{H}_{17} \mathrm{NO}_{4}$ & 305 & $\begin{array}{c}125-127 \\
(123-129) \\
{[14]}\end{array}$ & $305\left[\mathrm{M}^{+}\right]$ & 3407 & 1688 & 7.093 & 171.68 \\
\hline 6 & $2-\mathrm{OH}$ & $\mathrm{C}_{18} \mathrm{H}_{12} \mathrm{O}_{5}$ & 278 & $\begin{array}{c}150-152 \\
(153-156) \\
{[14]}\end{array}$ & $278\left[\mathrm{M}^{+}\right]$ & 3409 & 1686 & 7.349 & 171.61 \\
\hline 7 & $4-\mathrm{OH}$ & $\mathrm{C}_{18} \mathrm{H}_{12} \mathrm{O}_{5}$ & 278 & $\begin{array}{c}123-125 \\
(122-124) \\
{[14]}\end{array}$ & $278\left[\mathrm{M}^{+}\right]$ & 3410 & 1691 & 7.214 & 171.24 \\
\hline 8 & 4-OMe & $\mathrm{C}_{19} \mathrm{H}_{14} \mathrm{O}_{5}$ & 292 & $\begin{array}{c}145-146 \\
(147-150) \\
{[14]}\end{array}$ & $292\left[\mathrm{M}^{+}\right]$ & 3403 & 1682 & 7.008 & 170.16 \\
\hline 9 & 4-Me & $\mathrm{C}_{19} \mathrm{H}_{14} \mathrm{O}_{4}$ & 276 & $\begin{array}{c}123-124 \\
(119-122) \\
{[14]}\end{array}$ & $276\left[\mathrm{M}^{+}\right]$ & 3406 & 1684 & 7.114 & 170.96 \\
\hline 10 & $3-\mathrm{NO}_{2}$ & $\mathrm{C}_{18} \mathrm{H}_{11} \mathrm{NO}_{6}$ & 323 & $126-127$ & $323\left[\mathrm{M}^{+}\right]$ & 3415 & 1691 & 7.395 & 171.96 \\
\hline 11 & $4-\mathrm{NO}_{2}$ & $\mathrm{C}_{18} \mathrm{H}_{11} \mathrm{NO}_{6}$ & 323 & $\begin{array}{c}144-146 \\
(143-145) \\
{[14]}\end{array}$ & $323\left[\mathrm{M}^{+}\right]$ & 3416 & 1693 & 7.413 & 173.76 \\
\hline
\end{tabular}


Table 2. Results of statistical analysis of infrared $v\left(\mathrm{~cm}^{-1}\right) \mathrm{OH}$, CO stretches, NMR chemical shifts of $\mathrm{OH}$ and $\mathrm{CO}(\delta, \mathrm{ppm})$ of 3-hydroxy-2-(substituted phenyl)-4H-pyrano[3,2-b][1]benzofuron-4-ones with Hammett constants $\sigma, \sigma^{+}, \sigma_{\mathrm{I}}, \sigma_{\mathrm{R}}$ and $\mathrm{F}$ and R parameters.

\begin{tabular}{|c|c|c|c|c|c|c|c|}
\hline Frequency & Constants & $\mathbf{r}$ & I & $\rho$ & $\mathbf{s}$ & $\mathbf{n}$ & Correlated derivatives \\
\hline \multirow[t]{6}{*}{$v \mathrm{OH}$} & $\sigma$ & 0.998 & 3410.02 & 7.372 & 2.28 & 11 & $\begin{array}{c}\mathrm{H}, 3-\mathrm{Br}, 3-\mathrm{Cl}, 4-\mathrm{Cl}, 4-\mathrm{NMe}_{2}, \\
\text { 2-OH, 4-OH, 4-OMe, 4-Me, } \\
\text { 3-NO } \mathrm{NO}_{2}, 4-\mathrm{NO}_{2}\end{array}$ \\
\hline & $\sigma^{+}$ & 0.997 & 3411.18 & 4.223 & 2.63 & 11 & $\begin{array}{c}\mathrm{H}, 3-\mathrm{Br}, 3-\mathrm{Cl}, 4-\mathrm{Cl}, 4-\mathrm{NMe}_{2}, \\
\text { 2-OH, 4-OH, 4-OMe, 4-Me, } \\
\text { 3-NO } \mathrm{NO}_{2}, 4-\mathrm{NO}_{2}\end{array}$ \\
\hline & $\sigma_{\mathrm{I}}$ & 0.977 & 3406.39 & 13.103 & 2.69 & 11 & $\begin{array}{c}\mathrm{H}, 3-\mathrm{Br}, 3-\mathrm{Cl}, 4-\mathrm{Cl}, 4-\mathrm{NMe}_{2} \\
\text { 2-OH, 4-OH, 4-OMe, 4-Me, } \\
\text { 3-NO } \mathrm{NO}_{2}, 4-\mathrm{NO}_{2}\end{array}$ \\
\hline & $\sigma_{R}$ & 0.976 & 3412.65 & 9.870 & 3.39 & 11 & $\begin{array}{c}\mathrm{H}, 3-\mathrm{Br}, 3-\mathrm{Cl}, 4-\mathrm{Cl}, 4-\mathrm{NMe}_{2}, \\
\text { 2-OH, 4-OH, 4-OMe, 4-Me, } \\
\text { 3-NO } \mathrm{NO}_{2}, 4-\mathrm{NO}_{2}\end{array}$ \\
\hline & $\mathrm{F}$ & 0.967 & 3405.92 & 11.746 & 2.96 & 11 & $\begin{array}{c}\mathrm{H}, 3-\mathrm{Br}, 3-\mathrm{Cl}, 4-\mathrm{Cl}, 4-\mathrm{NMe}_{2}, \\
\text { 2-OH, 4-OH, 4-OMe, 4-Me, } \\
\text { 3- } \mathrm{NO}_{2}, 4-\mathrm{NO}_{2}\end{array}$ \\
\hline & $\mathrm{R}$ & 0.906 & 3412.77 & 7.081 & 3.31 & 11 & $\begin{array}{c}\mathrm{H}, 3-\mathrm{Br}, 3-\mathrm{Cl}, 4-\mathrm{Cl}, 4-\mathrm{NMe}_{2} \\
\text { 2-OH, 4-OH, 4-OMe, 4-Me, } \\
\text { 3- } \mathrm{NO}_{2}, 4-\mathrm{NO}_{2}\end{array}$ \\
\hline \multirow[t]{6}{*}{$v \mathrm{CO}$} & $\sigma$ & 0.905 & 1688.16 & 4.123 & 2.77 & 9 & $\begin{array}{c}\mathrm{H}, 3-\mathrm{Br}, 3-\mathrm{Cl}, 4-\mathrm{Cl}, 2-\mathrm{OH}, \\
\text { 4-OMe, 4-Me, 3- } \mathrm{NO}_{2}, 4-\mathrm{NO}_{2}\end{array}$ \\
\hline & $\sigma^{+}$ & 0.904 & 1688.77 & 2.101 & 2.99 & 9 & $\begin{array}{c}\mathrm{H}, 3-\mathrm{Br}, 3-\mathrm{Cl}, 4-\mathrm{Cl}, 2-\mathrm{OH}, \\
\text { 4-OMe, 4-Me, 3- } \mathrm{NO}_{2}, 4-\mathrm{NO}_{2}\end{array}$ \\
\hline & $\sigma_{\mathrm{I}}$ & 0.906 & 1685.88 & 8.119 & 2.74 & 10 & $\begin{array}{l}\mathrm{H}, 3-\mathrm{Br}, 3-\mathrm{Cl}, 4-\mathrm{Cl}, 4-\mathrm{NMe}_{2}, \\
\text { 2-OH, 4-OMe, 4-Me, 3- } \mathrm{NO}_{2}, \\
\text { 4-NO } \mathrm{NO}_{2}\end{array}$ \\
\hline & $\sigma_{\mathrm{R}}$ & 0.904 & 1689.76 & 6.118 & 2.99 & 9 & $\begin{array}{c}\mathrm{H}, 3-\mathrm{Br}, 3-\mathrm{Cl}, 4-\mathrm{Cl}, 4-\mathrm{NMe}_{2}, \\
\text { 2-OH, 4-OH, 4-Me, 3- } \mathrm{NO}_{2}, \\
\text { 4- } \mathrm{NO}_{2}\end{array}$ \\
\hline & $\mathrm{F}$ & 0.905 & 1685.51 & 8.741 & 2.78 & 10 & $\begin{array}{c}\mathrm{H}, 3-\mathrm{Br}, 3-\mathrm{Cl}, 4-\mathrm{Cl}, 4-\mathrm{NMe}_{2}, \\
\text { 2-OH, 4-OH, 4-Me, 3-NO } \\
\text { 4- } \mathrm{NO}_{2}\end{array}$ \\
\hline & $\mathrm{R}$ & 0.874 & 1689.62 & 3.704 & 3.12 & 11 & $\begin{array}{c}\mathrm{H}, 3-\mathrm{Br}, 3-\mathrm{Cl}, 4-\mathrm{Cl}, 4-\mathrm{NMe}_{2} \\
\text { 2-OH, 4-OH, 4-OMe, 4-Me, } \\
\text { 3- } \mathrm{NO}_{2}, 4-\mathrm{NO}_{2}\end{array}$ \\
\hline \multirow[t]{3}{*}{$\delta \mathrm{OH}$} & $\sigma$ & 0.804 & 7.171 & 0.141 & 0.13 & 11 & $\begin{array}{c}\mathrm{H}, 3-\mathrm{Br}, 3-\mathrm{Cl}, 4-\mathrm{Cl}, 4-\mathrm{NMe}_{2}, \\
\text { 2-OH, 4-OH, 4-OMe, 4-Me, } \\
\text { 3-NO } \mathrm{NO}_{2}, 4-\mathrm{NO}_{2}\end{array}$ \\
\hline & $\sigma^{+}$ & 0.884 & 7.192 & 0.077 & 0.13 & 11 & $\begin{array}{c}\mathrm{H}, 3-\mathrm{Br}, 3-\mathrm{Cl}, 4-\mathrm{Cl}, 4-\mathrm{NMe}_{2}, \\
\text { 2-OH, 4-OH, 4-OMe, 4-Me, } \\
\text { 3- } \mathrm{NO}_{2}, 4-\mathrm{NO}_{2}\end{array}$ \\
\hline & $\sigma_{\mathrm{I}}$ & 0.846 & 7.095 & 0.270 & 0.13 & 11 & $\begin{array}{c}\mathrm{H}, 3-\mathrm{Br}, 3-\mathrm{Cl}, 4-\mathrm{Cl}, 4-\mathrm{NMe}_{2}, \\
\text { 2-OH, 4-OH, 4-OMe, 4-Me, } \\
\text { 3- } \mathrm{NO}_{2}, 4-\mathrm{NO}_{2}\end{array}$ \\
\hline
\end{tabular}




\begin{tabular}{|c|c|c|c|c|c|c|c|}
\hline & $\sigma_{R}$ & 0.832 & 7.218 & 0.175 & 0.14 & 11 & $\begin{array}{c}\mathrm{H}, 3-\mathrm{Br}, 3-\mathrm{Cl}, 4-\mathrm{Cl}, 4-\mathrm{NMe}_{2}, \\
\text { 2-OH, 4-OH, 4-OMe, 4-Me, } \\
\text { 3- } \mathrm{NO}_{2}, 4-\mathrm{NO}_{2}\end{array}$ \\
\hline & $\mathrm{F}$ & 0.854 & 7.062 & 0.353 & 0.12 & 11 & $\begin{array}{c}\mathrm{H}, 3-\mathrm{Br}, 3-\mathrm{Cl}, 4-\mathrm{Cl}, 4-\mathrm{NMe}_{2}, \\
\text { 2-OH, 4-OH, 4-OMe, 4-Me, } \\
\text { 3- } \mathrm{NO}_{2}, 4-\mathrm{NO}_{2}\end{array}$ \\
\hline & $\mathrm{R}$ & 0.832 & 7.221 & 0.126 & 0.14 & 11 & $\begin{array}{l}\mathrm{H}, 3-\mathrm{Br}, 3-\mathrm{Cl}, 4-\mathrm{Cl}, 4-\mathrm{NMe}_{2} \\
\text { 2-OH, 4-OH, 4-OMe, 4-Me, } \\
\quad \text { 3- } \mathrm{NO}_{2}, 4-\mathrm{NO}_{2}\end{array}$ \\
\hline \multirow[t]{6}{*}{$\delta \mathrm{CO}$} & $\sigma$ & 0.829 & 171.15 & 0.690 & 1.08 & 11 & $\begin{array}{c}\mathrm{H}, 3-\mathrm{Br}, 3-\mathrm{Cl}, 4-\mathrm{Cl}, 4-\mathrm{NMe}_{2}, \\
\text { 2-OH, 4-OH, 4-OMe, 4-Me, } \\
\text { 3- } \mathrm{NO}_{2}, 4-\mathrm{NO}_{2}\end{array}$ \\
\hline & $\sigma^{+}$ & 0.819 & 171.41 & 0.273 & 1.11 & 11 & $\begin{array}{c}\mathrm{H}, 3-\mathrm{Br}, 3-\mathrm{Cl}, 4-\mathrm{Cl}, 4-\mathrm{NMe}_{2}, \\
\text { 2-OH, 4-OH, 4-OMe, 4-Me, } \\
\text { 3- } \mathrm{NO}_{2}, 4-\mathrm{NO}_{2}\end{array}$ \\
\hline & $\sigma_{\mathrm{I}}$ & 0.823 & 170.86 & 1.062 & 1.10 & 11 & $\begin{array}{c}\mathrm{H}, 3-\mathrm{Br}, 3-\mathrm{Cl}, 4-\mathrm{Cl}, 4-\mathrm{NMe}_{2}, \\
\text { 2-OH, 4-OH, 4-OMe, 4-Me, } \\
\text { 3- } \mathrm{NO}_{2}, 4-\mathrm{NO}_{2}\end{array}$ \\
\hline & $\sigma_{\mathrm{R}}$ & 0.833 & 171.49 & 1.347 & 1.07 & 11 & $\begin{array}{c}\mathrm{H}, 3-\mathrm{Br}, 3-\mathrm{Cl}, 4-\mathrm{Cl}, 4-\mathrm{NMe}_{2}, \\
\text { 2-OH, 4-OH, 4-OMe, 4-Me, } \\
\text { 3- } \mathrm{NO}_{2}, 4-\mathrm{NO}_{2}\end{array}$ \\
\hline & $\mathrm{F}$ & 0.832 & 170.65 & 1.614 & 1.07 & 11 & $\begin{array}{c}\mathrm{H}, 3-\mathrm{Br}, 3-\mathrm{Cl}, 4-\mathrm{Cl}, 4-\mathrm{NMe}_{2}, \\
\text { 2-OH, 4-OH, 4-OMe, 4-Me, } \\
\text { 3- } \mathrm{NO}_{2}, 4-\mathrm{NO}_{2}\end{array}$ \\
\hline & $\mathrm{R}$ & 0.822 & 171.40 & 0.652 & 1.11 & 11 & $\begin{array}{c}\mathrm{H}, 3-\mathrm{Br}, 3-\mathrm{Cl}, 4-\mathrm{Cl}, 4-\mathrm{NMe}_{2}, \\
\text { 2-OH, 4-OH, 4-OMe, 4-Me, } \\
\text { 3- } \mathrm{NO}_{2}, 4-\mathrm{NO}_{2}\end{array}$ \\
\hline
\end{tabular}

\section{1. Infrared spectral study}

Infrared spectra of all synthesized 3-hydroxy-2-(substituted phenyl)-4H-pyrano[3,2b][1]benzofuron-4-ones were recorded in $\mathrm{KBr}$ disc technique. From the spectra, the authors have expected the doublet for the carbonyl stretches $\left(\mathrm{cm}^{-1}\right)$ due to presence of cyclic $\alpha, \beta$ unsaturated moiety. But the doublet was not observed. It may happened by the suppression of hydroxyl group attached in $\alpha$ - position. The assigned $\nu \mathrm{CO}$ and $\mathrm{OH}$ stretches $\left(\mathrm{cm}^{-1}\right)$ were presented in Table 1. These data are correlated with Hammett substituent constants, $\mathrm{F}$ and $\mathrm{R}$ parameters by linear regression analysis. In the infrared spectral correlation, the Hammett equation was taken in the form as:

$$
v=\rho \sigma+v_{0}
$$

where $v_{0}$ is the frequency for the parent member of the series, $v$ is the frequency for the substituted member of the series, $\rho$ is the reaction constants and it depends on reaction conditions such as temperature and concentration, $\sigma$ is the substituent constants.

The results of statistical analysis are shown in Table 2. From Table 2, the $\mathrm{vOH}$ stretches $\left(\mathrm{cm}^{-1}\right)$ gave good correlation with Hammett $\sigma$ and $\sigma^{+}$constants. The Hammett $\sigma_{\mathrm{I}}, \sigma_{\mathrm{R}}$ substituent constants, $\mathrm{F}$ and $\mathrm{R}$ parameters have shown satisfactory correlation with $\mathrm{vOH}$ stretches $\left(\mathrm{cm}^{-1}\right)$ of 3-hydroxy-2-(substituted phenyl)-4H-pyrano[3,2-b][1]benzofuron-4-ones. All correlations gave positive $\rho$ values. This supports that the normal substituent effects 
operates in all systems. Similarly the multi-regression analysis gave good correlation with Swain-Lupton's [20] $\sigma_{\mathrm{I}}, \sigma_{\mathrm{R}}$ substituent constants, $\mathrm{F}$ and $\mathrm{R}$ parameters. The generated multiregression analysis equations are given in (2) and (3).

$$
\begin{aligned}
& \nu \mathrm{OH}\left(\mathrm{cm}^{-1}\right)=3408.44( \pm 1.764)+10.354( \pm 3.611) \sigma_{\mathrm{I}}+5.503( \pm 0.531) \sigma_{\mathrm{R}} \\
& (R=0.984, \mathrm{n}=11, P>95 \%) \\
& v \mathrm{OH}\left(\mathrm{cm}^{-1}\right)=3408.38( \pm 1.887)+10.817( \pm 3.901) \mathrm{F}+4.673( \pm 0.223) \mathrm{R} \\
& (R=0.983, \mathrm{n}=11, P>95 \%)
\end{aligned}
$$

The $v C O$ stretches $\left(\mathrm{cm}^{-1}\right)$ of 3-hydroxy-2-(substituted phenyl)-4H-pyrano[3,2-b][1] benzofuron-4-ones gave satisfactory correlations with Hammett substituent constants and $F$ parameter. The R parameter was failed in correlation. All correlations gave positive $\rho$ values.

This supports that the normal substituent effects operates in all systems. The failure in correlation for $\mathrm{R}$ parameters is due to the inability of transmittance of substituent effects of substituents on the $\mathrm{vCO}$ stretches and associated with the resonance conjugative structure as shown in Fig. 1.

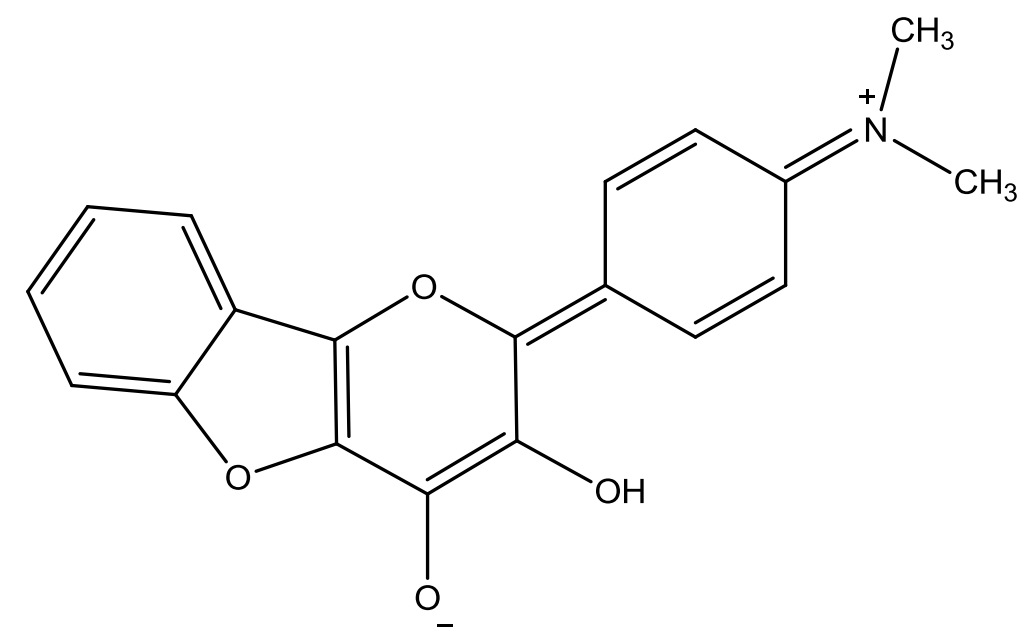

Figure. 1. The resonance-conjugative structure.

The $\mathrm{R}$ parameter was failed in single regression analysis. This is worthwhile, when seeking in the multi-regression analysis with Swain-Lupton's [20] $\sigma_{I}, \sigma_{R}$ substituent constants, $\mathrm{F}$ and $\mathrm{R}$ parameters. The generated multi-regression analysis equations are given in (4) and (5).

$$
\begin{gathered}
v \mathrm{CO}\left(\mathrm{cm}^{-1}\right)=1687.48( \pm 1.982)+6.414( \pm 4.057) \sigma_{\mathrm{I}}+3.441( \pm 1.378) \sigma_{\mathrm{R}} \\
(R=0.965, \mathrm{n}=11, P>95 \%) \\
v \mathrm{CO}\left(\mathrm{cm}^{-1}\right)=1686.59( \pm 2.142)+7.459( \pm 4.435) \mathrm{F}+2.046( \pm 1.125) \mathrm{R} \\
(R=0.962, \mathrm{n}=11, P>95 \%)
\end{gathered}
$$




\section{2. NMR spectral study}

The NMR chemical shifts $(\delta, \mathrm{ppm})$ of $\mathrm{OH}$ and $\mathrm{CO}$ protons and carbons of 3-hydroxy-2(substituted phenyl)-4H-pyrano[3, 2-b][1]benzofuron-4-ones were assigned and presented in Table 1 . These chemical shifts $(\delta, \mathrm{ppm})$ were correlated with Hammett substituent constants, $\mathrm{F}$ and $\mathrm{R}$ parameters using single and multi-parameter correlations. In this NMR spectral correlation, the Hammett equation was employed as,

$$
\delta=\rho \sigma+\delta_{\mathrm{o}}
$$

where $\delta_{\mathrm{o}}$ is the chemical shift $(\delta, \mathrm{ppm})$ for the parent member of the series, $\delta$ is the chemical shift $(\delta, \mathrm{ppm})$ for the substituted member of the series, $\rho$ is the reaction constants and it depends on reaction conditions such as temperature and concentration, $\sigma$ is the substituent constants.

The results of statistical analyses were presented in Table 2. From the Table 2, the correlation of $\mathrm{OH}$ and $\mathrm{CO}$ protons and carbon chemical shifts $(\delta$, ppm) of 3-hydroxy-2(substituted phenyl)-4H-pyrano[3,2-b][1] benzofuron-4-ones were failed with Hammett substituent constants, $\mathrm{F}$ and $\mathrm{R}$ parameters. All correlations gave positive $\rho$ values. This supports that the normal substituent effects operates in all systems. The reason for failure of correlation was stated earlier and associated with resonance conjugative structure as shown in Fig. 1. In view of the inability of prediction of effect of substituents on the $\mathrm{OH}$ and $\mathrm{CO}$ protons and carbon chemical shifts $(\delta, \mathrm{ppm})$, they are worthwhile and produce satisfactory correlations when seeking in multi-regression analysis with Swain-Lupton's [20] $\sigma_{\mathrm{I}}, \sigma_{\mathrm{R}}$ substituent constants, $\mathrm{F}$ and $\mathrm{R}$ parameters. The generated multi-regression analysis equations are given in (7-10)

$$
\begin{gathered}
\delta \mathrm{OH}(\mathrm{ppm})=7.124( \pm 0.100)+0.321( \pm 0.021) \sigma_{\mathrm{I}}+0.078( \pm 0.001) \sigma_{\mathrm{R}} \\
(R=0.974, \mathrm{n}=11, P>95 \%) \\
\delta \mathrm{OH}(\mathrm{ppm})=7.091( \pm 0.092)+0.319( \pm 0.021) \mathrm{F}+0.055( \pm 0.012) \mathrm{R} \\
(R=0.955, \mathrm{n}=11, P>95 \%) \\
\delta \mathrm{CO}(\mathrm{ppm})=171.29( \pm 0.812)+0.491( \pm 0.066) \sigma_{\mathrm{I}}+1.142( \pm 0.152) \sigma_{\mathrm{R}} \\
(R=0.934, \mathrm{n}=11, P>95 \%) \\
\delta \mathrm{CO}(\mathrm{ppm})=170.83( \pm 0.852)+1.405( \pm 0.176) \mathrm{F}+0.338( \pm 0.105) \mathrm{R} \\
(R=0.934, \mathrm{n}=11, P>95 \%)
\end{gathered}
$$

\section{CONCLUSION}

Totally eleven benzofuranyl flavonols [3-hydroxy-2-(substituted phenyl)- $4 \mathrm{H}$ pyrano[3,2-b][1]benzofuron-4-ones] have been prepared by cyclization of 3hydroxybenzofuranyl chalcones with $30 \%$ hydrogen peroxide in the presence of sodium bicarbonate. The synthesized flavonols were characterized by their physical constants, analytical and spectroscopic data. The infrared spectral $v \mathrm{OH}, \mathrm{CO}$ stretches $\left(\mathrm{cm}^{-1}\right), \mathrm{NMR}$ chemical shifts of $\mathrm{OH}, \mathrm{CO}(\delta, \mathrm{ppm})$ proton and carbons of these flavonols were assigned and correlated with Hammett substituent constants, F and R parameters using single and multi 
linear regression analysis. From the results of statistical analyses, $v \mathrm{OH}, \mathrm{CO}$ stretches $\left(\mathrm{cm}^{-1}\right)$, gave good and satisfactory correlation in single and multi-regression analysis.

The NMR chemical shifts of $\mathrm{OH}, \mathrm{CO}(\delta, \mathrm{ppm})$ proton and carbons of these flavonols were failed in single parameter correlation and satisfactory in multi-parameter correlation.

\section{References}

[1] G. Thirunarayana, P. Ananthakrishna Nadar, Asian J. Chem. 14(3-4) (2002) 1518-1522.

[2] W. F. Winecoff, D. W. Boykin Jr., J. Org. Chem. 37 (4) (1972) 676.

[3] A. Perjessy, M. Laucova, Coll. Czech. Chem. Commun. 36 (1971) 2944-22950.

[4] T. A. Foglia, P. A. Barr, M. J. Idacavage, J. Org. Chem. 41 (1976) 3452-3455.

[5] (a) V. Všetečka, J. Pecka and M. Procházka, Collect. Czech. Chem. Commun. 47 (1982) 277-285.

(b) G. Thirunarayanan, G. Vanangamudi, V. Sathiyendiran, K. Ravi, Indian J. Chem. 50B(4) (2011) 593-604.

[6] G. Thirunarayanan, M. Gopalakrishnan, G. Vanangamudi, Spectrochim. Acta. 67A (2007) 1106-1612.

[7] G. Thirunarayanan, K. G. Sekar, J. Taibah Univ. Sci. 2013. DOI: 10.1016/j.jtusci.2013.11.003.

[8] S. John Joseph, R. Arulkumaran, D. Kamalakkannan, S. P. Sakthinathan, R. Sundararajan, R. Suresh, S. Vijayakumar, K. Ranganathan, N. Kalyanasundaram, G. Vanangamudi, G. Thirunarayanan, International Letters of Chemistry, Physics and Astronomy 4 (2014) 48-65.

[9] G. Thirunarayanan, International Letters of Chemistry, Physics and Astronomy 5 (2014) 89-98.

[10] S. John Joseph, D. Kamalakkannan, R. Arulkumaran, S. P. Sakthinathan R. Suresh, R. Sundararajan, S. Vijayakumar, K. Ranganathan, G. Vanangamudi, G. Thirunarayanan, International Letters of Chemistry, Physics and Astronomy 5 (2014) 99-123.

[11] K. G. Sekar, G. Thirunarayanan, International Letters of Chemistry, Physics and Astronomy 8(2) (2013) 160-174.

[12] G. Thirunarayanan, International Letters of Chemistry, Physics and Astronomy 4 (2014) 109-116.

[13] D. Kamalakkannan, G. Vanangamudi, R. Arulkumaran, K. Thirumurthy, P. Mayavel, G. Thirunarayanan, Elixir Org. Chem. 46 (2012) 8157-8166.

[14] P. M. G. Samy, Y. R. Prasad, B. S. Sastry, D. Giles, M. Gurumurthy, Y. S. Agasimundin, Der Pharm. Chem 5 (2013) 8-13.

[15] M. Subramanian, G. Vanangamudi, G. Thirunarayanan, Spectrochim Acta 110A (2013) 116-123.

[16] K. G Sekar, G. Thirunarayanan, International Letters of Chemistry, Physics and Astronomy 8(3) (2013)249-258. 
[17] K. Sathiyamoorthy, V. Mala, S.P. Sakthinathan, D. Kamalakkannan, R. Suresh, G. Vanangamudi, G. Thirunarayanan, Spectrochim. Acta 112A (2013) 245-256.

[18] S. P. Sakthinathan, R. Suresh, V. Mala, K. Sathiyamoorthy, D. Kamalakkannan, K. Ranganathan, S. John Joseph, G. Vanangamudi, G. Thirunarayanan, Int. J. Sci. Res. Know. 1(11) (2013) 472-483.

[19] G. Thirunarayanan, M. Suresh, International Letters of Chemistry, Physics and Astronomy 4 (1) (2014) 1-11.

[20] C. G. Swain, E. C. Lupton Jr., J. Am. Chem. Soc. 90 (1968) 4328-4337. 